\title{
Integrated pre-concentrator gas sensor micro system for trace gas detection
}

\author{
Martin Leidinger ${ }^{1}$, Tilman Sauerwald ${ }^{1}$, Max Rieger ${ }^{2}$, Christine Alépée ${ }^{3}$, Andreas Schütze $^{1}$ \\ ${ }^{1}$ Saarland University, Lab for Measurement technology, Saarbrücken/Germany \\ m.leidinger@Imt.uni-saarland.de \\ ${ }^{2}$ Fraunhofer Institute for Chemical Technology ICT, Pfinztal/Germany \\ ${ }^{3}$ SGX Sensortech SA, Corcelles/Switzerland
}

\begin{abstract}
An integrated microsystem for trace gas detection is presented. Using metal-organic frameworks for gas pre-concentration and metal oxide semiconductor gas sensors for gas measurement, ppb concentrations of benzene have successfully been detected. Two metal organic framework materials have been characterized regarding their performance for gas adsorption and desorption. A state-ofthe-art commercial adsorbent material was also characterized via inverse gas chromatography (iGC) for reference, however it was outperformed by the metal-organic frameworks.

The materials were deposited on micro hot plates and integrated into sensor packages together with dual metal oxide semiconductor gas sensors. These integrated systems have been characterized in FEM simulations as well as test measurements. Both the simulations and the measurements prove the suitability of the design of the system. Significant increase of ppb level target gas concentrations inside the systems could be observed with the gas sensors during thermal desorption of the preconcentrator.
\end{abstract}

Key words: Gas pre-concentration, metal organic frameworks, inverse gas chromatography, integrated sensor system, metal oxide semiconductor gas sensor.

\section{Introduction}

For evaluation of indoor air quality (IAQ), several gaseous compounds must be taken into account especially a variety of volatile organic compounds (VOCs). These compounds can have negative health effects already at very low concentrations in the ppb range. Based on toxicity and prevalence, according to the World Health Organization (WHO), the highest priority VOCs are formaldehyde, benzene and naphthalene [1]. The respective guideline threshold values for these substances in indoor air are $0.1 \mathrm{mg} / \mathrm{m}^{3}$ (81 ppb) for formaldehyde [1] $0.01 \mathrm{mg} / \mathrm{m}^{3}$ (1.9 ppb) for naphthalene [1] and $5 \mu \mathrm{g} / \mathrm{m}^{3}$ (1.6 ppb) for benzene [2].

In order to be able to reliably detect such small target compound concentrations, gas preconcentration can be applied. Using a suitable material with a high surface area, gases can be collected over a certain period of time and then thermally desorbed, which generates a short pulse of high gas concentration.

Measurement of this gas pulse can be performed with metal oxide semiconductor (MOS) gas sensors in temperature cycled operation (TCO), which has been shown to be suitable to detect VOCs in the ppb range [3]. As pre-concentrator materials, meta-organic frameworks (MOFs) can be applied. These materials have a very high surface area (in some cases more than $1000 \mathrm{~m}^{2} / \mathrm{g}$ ) which means relatively large quantities of gas can be adsorbed and stored in a small volume of the material. MOFs are a novel substance class of highly porous coordination polymers (PCPs) with extraordinary properties. They are composed of metal- or metal-cluster-cations (so called "nodes") and multidentate anionic or neutral organic molecules (so called "linkers") [4]. The resulting crystalline 3-dimensional network exhibits pores and channels with spatial and chemical uniformity. Pore sizes up to almost $98 \AA$ have been reported [5]. Optimizing synthesis conditions (choice of linkers, nodes and their molar ratios) it is possible to tailor a porous crystal for a specific need. This may include gas storage, separation as well as catalysis, sensing and drug delivery. Recently published computational investigations suggest their usage as preconcentrator material for small molecules such as xylenes, phosphonates and high explosives (TNT) [6-9].

In our investigation, two types of MOFs have 
been characterized regarding their benzene adsorption potential at room temperature and their desorption characteristics at elevated temperature. Based on these results, an integrated device with a pre-concentrator and a dual metal oxide gas sensor was developed. The pre-concentrator material was deposited on a MEMS hot plate, which allows for fast temperature changes of the MOF. The physical properties of this system, e.g. gas transport and gas enrichment, have been simulated.

With the first prototypes of integrated systems, test measurements with benzene have been performed, which show the basic functionality of the integrated approach. The pre-concentration effect can clearly be observed in the gas sensor signals.

\section{MOFs as pre-concentrator materials}

Metal-organic frameworks and Tenax ${ }^{\circledR}$ TA were obtained from Sigma Aldrich. Two commercially available so-called Basolites $\AA$, A100 or MIL-53, and C300 or HKUST-1 were tested in comparison with a standard adsorbent (Tenax® TA in 60-80). The MOF powders showed volume weighted particle diameter means $(D[4,3])$ of $16,2 \mu \mathrm{m}$ and $19,8 \mu \mathrm{m}$ for HKUST-1 and MIL-53, respectively.

Inverse gas chromatography (iGC) [10] was used in order to evaluate affinities of benzene towards MOFs and Tenax ${ }^{\circledR}$ TA. A fixed bed (about $10 \mathrm{mg}$ ) of sorbent powder was inserted in a gas chromatograph (GC) and eluted with nitrogen as carrier gas. A chromatogram was recorded doing multiple injections of gaseous benzene at different temperatures. Retention volumes are calculated by subtracting the dead time (methane) and multiplying carrier gas flows (usually $10 \mathrm{ml} / \mathrm{min}$ via gas flow meter) with retention times. They are normalized in terms of dividing them by sorbent masses, see Fig. 1 .

These values indicate relative affinities of guest molecules towards respective sorbent materials. This process, however, is highly dynamic and cannot be directly compared to static saturation as in terms of preconcentration. However, it can be used in order to estimate minimum adsorption and maximum desorption intervals. Furthermore it shows that both MOFs have a higher tendency to adsorb benzene compared to Tenax® TA. This may be attributed to their porosity as well as to chemical interaction within their internal surface.

By immersing bulk MOF powders in different solvents, it was possible to create stable - in terms of sedimentation - suspensions.

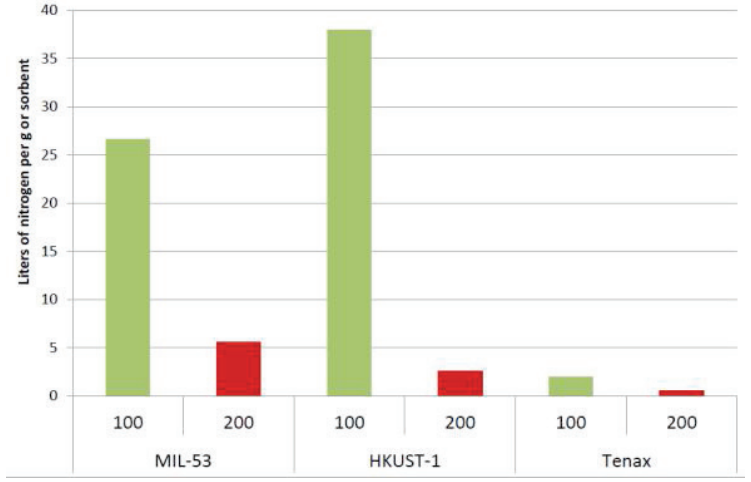

Fig. 1. Normalized retention volumes for benzene on 2 different MOFS (MIL-53 and HKUST-1) and Tenax ${ }^{\circledR}$ TA. Values in liters of nitrogen needed to elute benzene from a fixed bed of sorbent. Temperatures in ${ }^{\circ} \mathrm{C}$.

These were applied to alumina heater substrates $\left(3 * 3 \mathrm{~mm}^{2}\right)$ using capillaries and tipping them manually. Di-n-butylether finally became the solvent of choice. Amounts of approximately $50 \mathrm{mg}$ of MOF were applied to the substrates.

Gas samples were supplied in gas sampling bags (Supelco, 30240-U). A T-valve was used to switch between zero air and analyte in zero air. Selected gas streams were conducted through a stainless steel measurement chamber containing pre-concentrators (PCs). To measure the pre-concentration efficiency of the film we used a setup presented in [11], where the gas stream exiting the measurement chamber was monitored online by a mass spectrometer (Pfeiffer GSD 301 quadrupole MS) using multiple ion detection and regulated using a mass-flow controller and a vacuum pump. The mass detector's linearity was evaluated measuring and further diluting various gas sampling bags by a factor of 10 . Linearity was observed for a range from $100 \mathrm{ppb}$ to $300 \mathrm{ppm}$.

Figs. 2 and 3 show different series of peaks by adsorbing analytes in carrier gas at room temperature for different times. Desorption was done by heating the preconcentrator to $200{ }^{\circ} \mathrm{C}$. Ion currents are normalized with respect to their mass channels value for zero air. Desorption peaks maximum values are also normalized in this fashion. In case of $10 \mathrm{ppm}$ of benzene within the carrier gas (Fig. 3), it was possible to obtain an enrichment factor of almost $700 \%$ using 60 minutes of adsorption before thermal desorption and $550 \%$ in case of $30 \mathrm{~min}$. In case of $1 \mathrm{ppm}$ (Fig. 2) this value drops to $460 \%$ for $60 \mathrm{~min}$. Lower adsorption intervals $(30,15$ and $10 \mathrm{~min}$ ) lead to preconcentration factors of 310 , 210 and $190 \%$, respectively. 


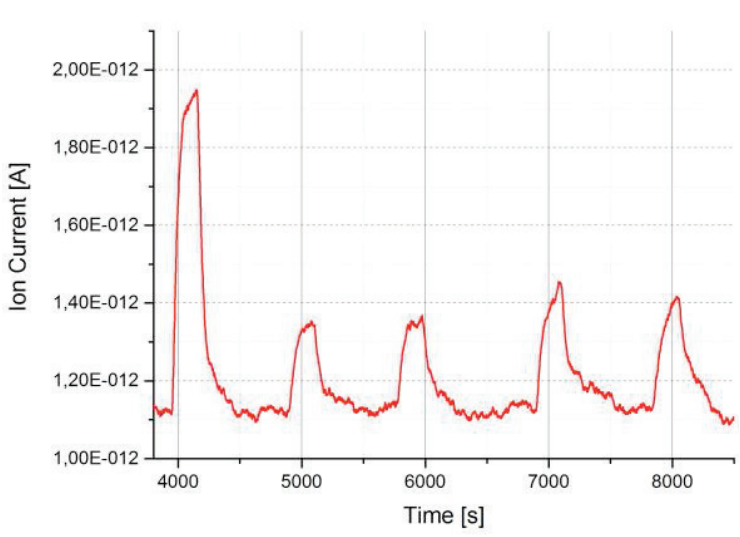

Fig. 2. Sequence of peaks from different adsorption intervals of $1 \mathrm{ppm}$ of benzene in zero-air (left to right in minutes):60, 10, 10, 15, 10.MOF: HKUST-1

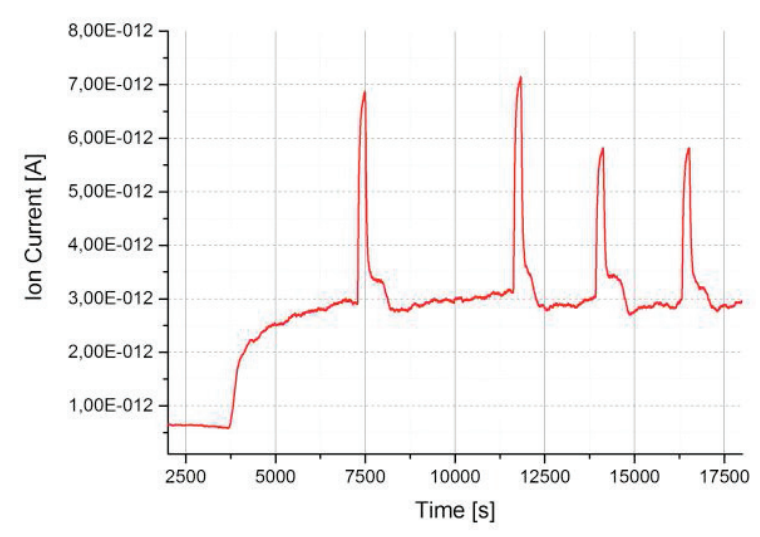

Fig. 3. Sequence of peaks from different adsorption intervals of $10 \mathrm{ppm}$ of benzene in zero-air (left to right in minutes):60, 60, 30, 30.MOF: HKUST-1.

\section{Integrated pre-concentrator gas sensor system}

A pre-concentrator chip with the MOF material deposited on a micro hot plate has been integrated into a ceramic SMD package in addition to a commercial dual $\mathrm{WO}_{3}$ gas sensor chip (SXG Sensortech SA). The external dimensions of the package are $5 \times 7 \times 1 \mathrm{~mm}$. The geometry of the setup was implemented into COMSOL Multiphysics for FEM simulations of the behavior of the system regarding gas diffusion and adsorption / desorption of gas at the pre-concentrator. Fig. 4 shows the 2D geometry model. As can be seen in the COMSOL side view, gas access from the environment into the package is possible only through a narrow orifice above the preconcentrator chip.

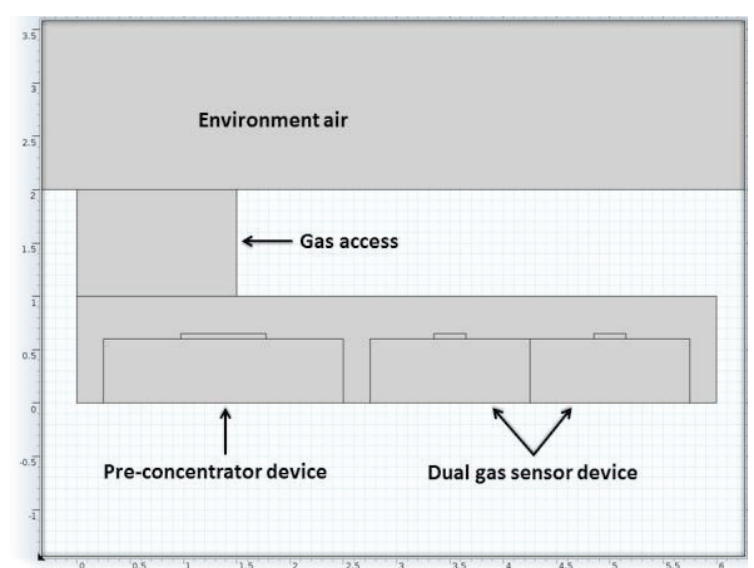

Fig. 4. $2 D$ model of the integrated system containing a pre-concentrator chip and the dual gas sensor chip. Gas access to the environment is limited to a narrow access hole.

The width of this gas access is $1.5 \mathrm{~mm}$ in this case. Because of this geometry, the preconcentrator is close to the environmental air, which contains the gases to be collected and measured. The second effect of this is that the pre-concentrator adsorbs the gas from inside the package, which leads to a very low gas concentration at the gas sensors.

The pre-concentrator has been defined with a length of $800 \mu \mathrm{m}$ and a thickness of $50 \mu \mathrm{m}$. A variable partition coefficient regarding the gas species at its geometry walls was introduced. The partition coefficient is given by the density of molecules in air (mobile phase) divided by the density in the pre-concentrator (stationary phase). It can be estimated from the vapor pressure of the compound. Using these settings, gas diffusion inside the system and gas exchange with the environment has been simulated. Simulated operation mode of the system was the following. At the start of the simulation run, the gas concentration in the air (environment as well as package) was set to $4^{*} 10^{-7} \mathrm{~mol} / \mathrm{m}^{3}$, or approx. $10 \mathrm{ppb}$. Due to the partition coefficient at the air / pre-concentrator barrier, gas is adsorbed by the pre-concentrator material. After $600 \mathrm{~s}$ of adsorption, the concentration distribution is that shown in Fig. 5 left. Nearly all gas from inside the package has been adsorbed by the pre-concentrator; the gas concentration at the sensors is very low. Gas from the environment is being adsorbed.

At $t=600 \mathrm{~s}$ in the simulation, the preconcentrator is switched from adsorption to desorption, simply by increasing the partition coefficient by a factor of 1000 , which is simulating an increase of temperature in the order of $200^{\circ} \mathrm{C}$. 

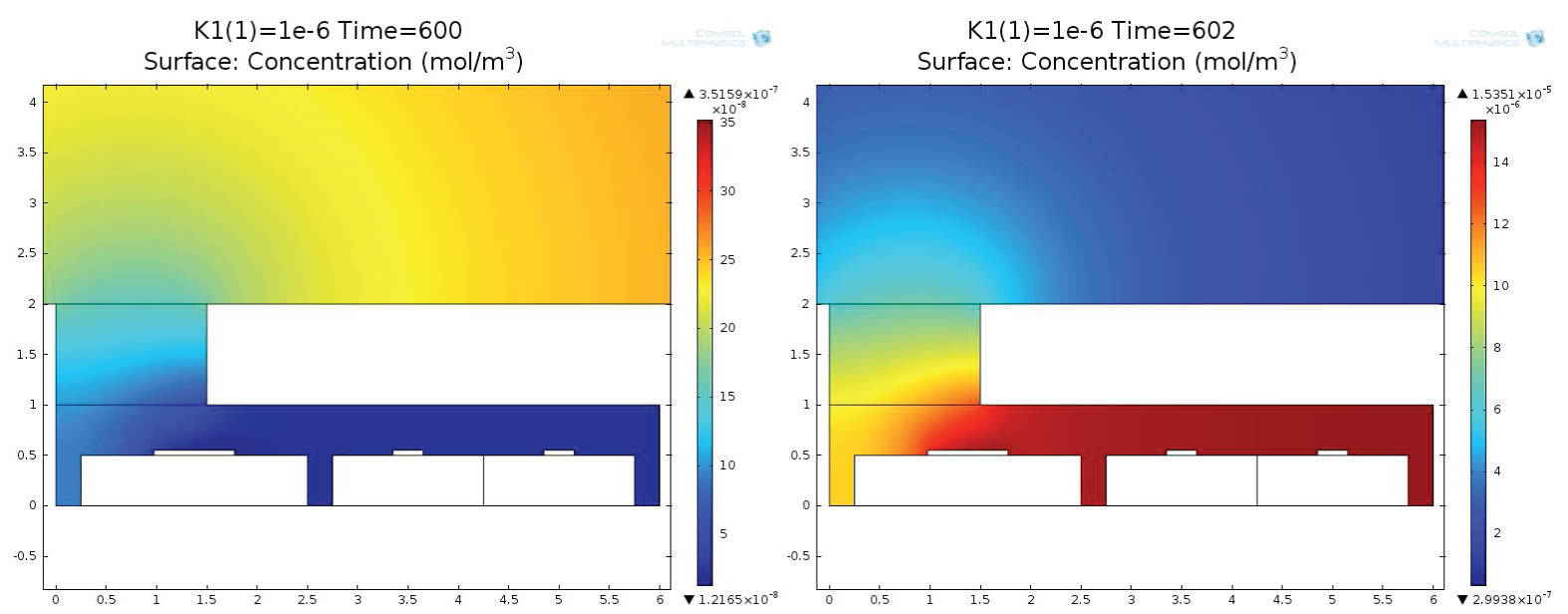

Fig. 5. Simulated gas distribution inside the system after $600 \mathrm{~s}$ of adsorption (left) and $2 \mathrm{~s}$ after beginning of desorption (right).
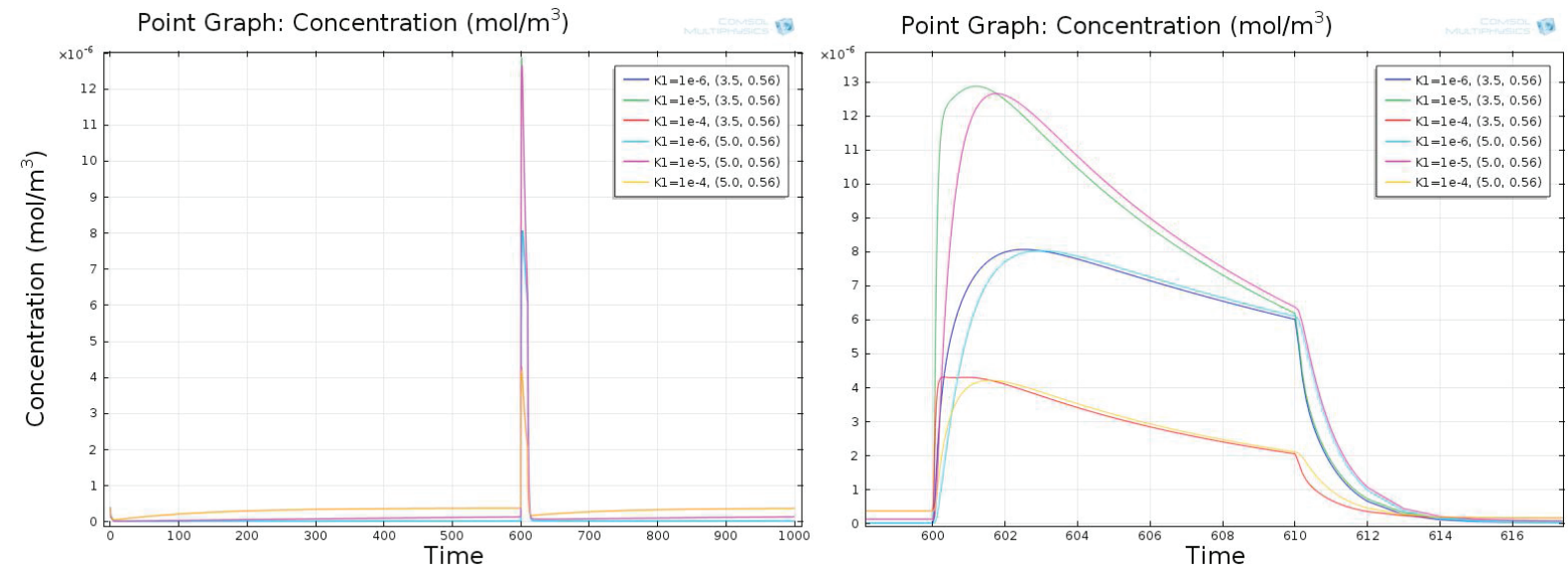

Fig. 6. Simulated gas concentrations at the two sensors for three different pre-concentrator partition coefficients during an adsorption / desorption period.

The condition of the gas $2 \mathrm{~s}$ after start of desorption is shown in Fig 5 right. The highest gas concentration (red), in this case approx. $1.5^{*} 10^{-5} \mathrm{~mol} / \mathrm{m}^{3}$ is at the area where the gas sensors are placed.

The gas concentrations just above the surfaces of the centers of the two gas sensors during an adsorption / desorption period are plotted in Fig 6 left, a detail of the desorption phase is given in Fig 6 right. Three different partition coefficients were compared in this simulation $\left(10^{-4}, 10^{-5}, 10^{-6}\right)$, as the coefficient is depending on the gas.

The results show that the gas takes about $2 \mathrm{~s}$ to to be transported from the pre-concentrator to the gas sensors. For lower volatile substances (low partion coefficient) the transport effectively slower, since the desorption takes a longer time.

The maximum concentration is obtained with a partition coefficient of $10^{-5}$. At the higher partition coefficient $\left(10^{-4}\right)$, i.e. a volatile gas, the signal is low because not much gas is adsorbed at the preconcentrator. At the lowest coefficient $\left(10^{-6}\right)$, i.e. a non-volatile species, desorption of the gas is incomplete, hence the lower signal compared to the middle partition coefficient of $10^{-5}$. This value generates the highest gas concentration at the sensors, as a lot of gas is adsorbed and also desorbed.

The gas concentration is increased by a factor of 32 (partition coefficient of $\left.10^{-5}\right), 20\left(10^{-6}\right)$ or $11\left(10^{-4}\right)$.

\section{Test measurement and results}

Initial test measurements have been performed with an integrated microsystem. As test gas, benzene was applied, in concentrations of $1 \mathrm{ppm}, 100 \mathrm{ppb}$ and $10 \mathrm{ppb}$. The operation modes of the sensor and the pre-concentrator are displayed in Fig. 7. For $298 \mathrm{~s}$ resp. $300 \mathrm{~s}$, both devices were switched off. The preconcentrator was switched off for best adsorption performance, the sensors were 
switched off to prevent thermal crosstalk which could heat up the MOF material. $298 \mathrm{~s}$ into the cycle, $2 \mathrm{~s}$ before the pre-concentrator is heated, the sensors were switched on by heating them to $400{ }^{\circ} \mathrm{C}$ for $2 \mathrm{~s}$ for cleaning their surfaces and then set to $300{ }^{\circ} \mathrm{C}$ for gas detection. At $300 \mathrm{~s}$, the pre-concentrator is set to $200{ }^{\circ} \mathrm{C}$. The MOF was heated for $3 \mathrm{~min}$, then the device was switched off again. The sensors were heated for two more minutes. The length of a full cycle was $600 \mathrm{~s}$ or $10 \mathrm{~min}$.

The system was operated in zero air and in zero air with the three benzene concentrations added. For signal processing, the relative change of conductance $\mathrm{G} / \mathrm{G}_{0}$ across a full cycle was calculated for each benzene concentration. The results for two target gas concentrations for the sensor further away from the preconcentrator are shown in Fig. 8. During the adsorption phase, there is no signal; the calculated quotient is quite noisy as the sensor signals are noisy when the sensors are switched off. When the MOF heater is switched on, the sensor signal starts to rise, and reaches its peak after about $2 \mathrm{~s}$. This is the time the simulation calculated for the maximum of the gas concentration at the sensor. After approx. $15 \mathrm{~s}$ the normalized signal is stable.

By calculating $G / G_{0}$, all effects that do not result from the benzene, like the sensor conductance behavior at heating up and cooling down or thermal crosstalk, should be suppressed. This means that the signals result only from the benzene.

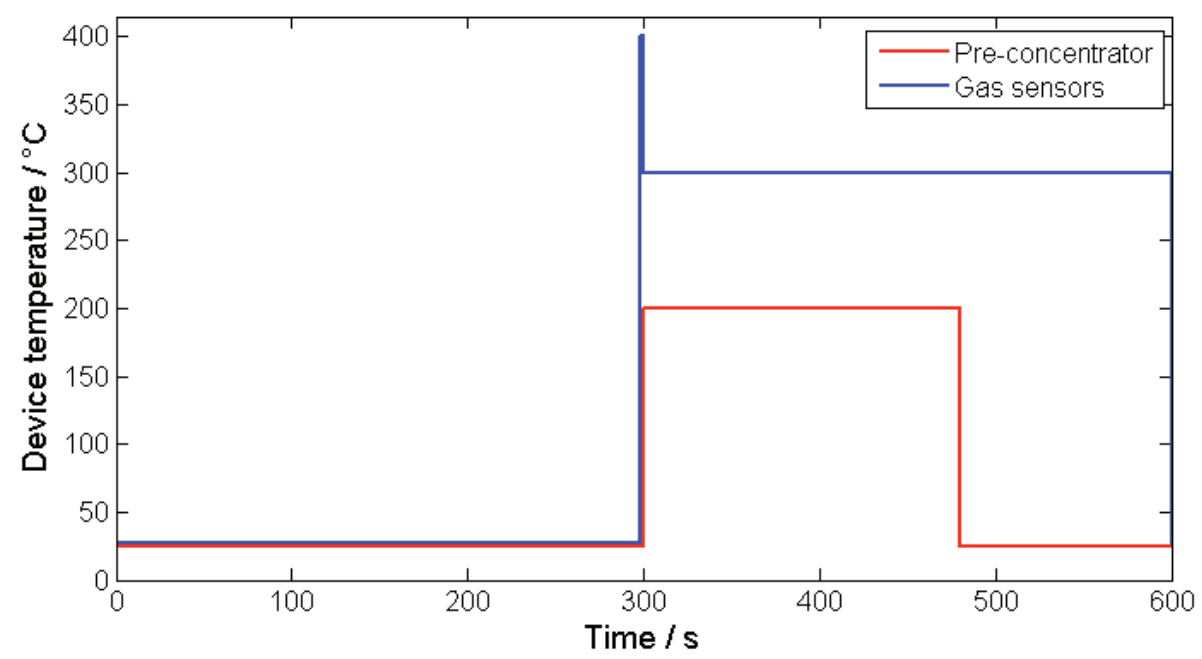

Fig. 7. Programmed temperature profiles of the pre-concentrator (red) and the MOS gas sensors (blue)
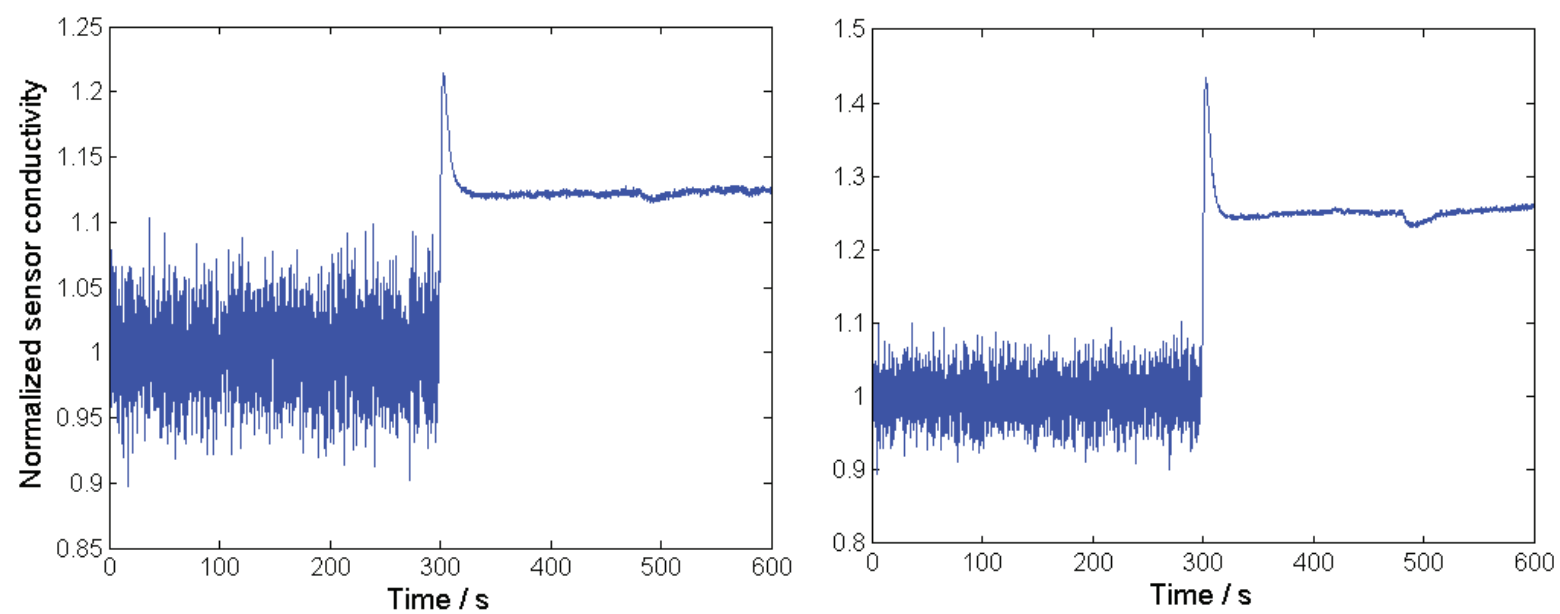

Fig. 8. Normalized sensor conductances $\mathrm{G} / \mathrm{G}_{0}$ for a cycle at $10 \mathrm{ppb}$ of benzene (left) and one at $100 \mathrm{ppb}$ of benzene (right) 
The shape of the peak corresponds very well to the results of the simulations, it can be assumed that this is caused by the desorption of the pre-concentrator. The signal peak generated by the gas desorption at $10 \mathrm{ppb}$ of benzene is nearly as high as the steady sensor signal after the peak at $100 \mathrm{ppb}$. This suggests that a gas pre-concentration of up to the factor of nearly 10 can be achieved.

\section{Conclusions}

An integrated micro pre-concentrator gas sensor system has been designed, simulated, realized and tested. The simulations show that the chosen design is suitable for the task of a high temporal gas concentration increase at the gas sensors.

The suitability of the metal-organic framework materials has been tested via invers gas chromatography measurements for adsorption and mass spectrometry measurements for desorption. Using iGC, relative affinities of benzene towards MOFs and a state-of-the-art adsorbent (Tenax $\left.{ }^{\circledR} \mathrm{TA}\right)$ could be determined. The MOFs outperformed Tenax $®$ in this setup and are suitable for pre-concentrating benzene. In a dynamic adsorption desorption process benzene preconcentration factors using MOFs could be determined and depend on the initial concentration of benzene.

A FEM model of the integrated system has been created and the gas diffusion inside the system and its interaction with the environment have been simulated. Performance of the system is strongly influenced by the partition coefficient of the gas at the surface of the preconcentrator material, which is depending on both the gas and the porous layer. This parameter determines the gas concentration inside the package at desorption but also the time from start of desorption until the gas has reached the sensors. In the simulations, the initial gas concentration could be increased by a factor of 11 to 32 , depending on the partition coefficient.

With the integrated system, test gas measurements for benzene pre-concentration have been successfully performed. For $10 \mathrm{ppb}$ of benzene, the sensor signal was nearly at the level of $100 \mathrm{ppb}$ without pre-concentration.

The performance of the system will be further enhanced by increasing the MOF coated area and by improving the operation modes of the devices.

\section{References}

[1] World Health Organization: WHO Guidelines for Indoor Air Quality: Selected Pollutants, Geneva (2010)

[2] European Parliament, Council of the European Union: Directive 2008/50/EC of the European Parliament and of the Council of 21 May 2008 on ambient air quality and cleaner air for Europe, Official Journal of the European Union, Volume 51 (2008)

[3] Leidinger, M., Sauerwald, T., Reimringer, W., Ventura, G., and Schütze, A.: Selective detection of hazardous VOCs for indoor air quality applications using a virtual gas sensor array, J. Sens. Sens. Syst., 3 (2014), pp. 253-263, doi:10.5194/jsss-3-253-2014

[4] Rowsell, J. L. C.; Yaghi, O. M. (2004). Metalorganic frameworks: a new class of porous materials. Microporous and Mesoporous Materials, 73, 3-14.

[5] Deng, H.; Grunder, S.; Cordova, K. E.; Valente, C.; Furukawa, H.; Hmadeh, M.; Gandara, F.; Whalley, a. C.; Liu, Z.; Asahina, S.; Kazumori, H.; O'Keeffe, M.; Terasaki, O.; Stoddart, J. F.; Yaghi, O. M. (2012). Large-Pore Apertures in a Series of Metal-Organic Frameworks. Science, 336, 10181023.

[6] Greathouse, J. A.; Ockwig, N. W.; Criscenti, L. J.; Guilinger, T. R.; Pohl, P.; Allendorf, M. D. (2010). Computational screening of metal-organic frameworks for large-molecule chemical sensing. Physical Chemistry Chemical Physics : PCCP, 12, 12621-12629.

[7] Asha, K. S., Bhattacharyya, K. \& Mandal, S. Discriminative detection of nitro aromatic explosives by a luminescent metal-organic framework. J. Mater. Chem. C 2, 10073-10081 (2014).

[8] Lan, A. et al. A luminescent microporous metalorganic framework for the fast and reversible detection of high explosives. Angew. Chem. Int. Ed. Engl. 48, 2334-8 (2009).

[9] Yamagiwa, H. et al. Detection of Volatile Organic Compounds by Weight-Detectable Sensors coated with Metal-Organic Frameworks. Sci. Rep. 4, 6247 (2014).

[10] Schneider, M., Goss, K. U. (2009). Systematic investigation of the sorption properties of tenax TA, chromosorb 106, porapak n, and carbopak $\mathrm{f}$. Analytical Chemistry, 81(8), 3017-3021. doi:10.1021/ac802686p

[11] M. Leidinger, M. Rieger, T. Sauerwald, M. Nägele, J. Hürttlen, A. Schütze, Trace gas VOC detection using metal-organic frameworks micro pre-concentrators and semiconductor gas sensors, 